\title{
Cultura y democratización en Chile. Los nuevos desafíos
}

\section{Culture et démocratisation au Chili. Les nouveaux défis}

\author{
Manuel Antonio Garretón M. ${ }^{1}$ \\ Doctor en Sociología, Profesor de la Universidad de Chile \\ Miembro Asociado del Centre d'Analyse et Intervention Sociologiques, EHESS/ \\ CNRS, Paris \\ Premio Nacional de Humanidades y Ciencias Sociales \\ magarret@uchile.cl
}

\begin{abstract}
Resumen
Este texto, emanado de una conferencia inaugural, dibuja los contornos del terreno en que se aloja el debate actual sobre la "cultura" en Chile. Analizando los principales elementos que sostienen -y tensionan- el cuadro institucional, político, económico y social del país, ofrece una radiografía de las condiciones de emergencia del fenómeno que abordó el Coloquio. La relación entre cultura y democratización es tratada aquí desde una mirada compleja que cruza institucionalidad y sustrato: soporte y contenido.

Se intenta así esbozar un marco sociopolítico y cultural para los análisis de la edición independiente que esta publicación ofrece, a partir del principio que la producción cultural, todo lo contrario de ser un ámbito aislado de la sociedad, constituye un elemento indispensable y central de la vida democrática.
\end{abstract}

Palabras Clave: Cultura, democratización política, actores culturales, transformaciones culturales.

\section{Résumé}

Lauteur dessine dans ce texte, prononcé lors de la conférence inaugurale de ces journées détude, les contours du débat actuel sur la question de la «culture» au Chili. Il analyse les principaux éléments formant le cadre institutionnel, politique, économique et social de ce pays ainsi que les tensions qui le traversent, et propose

1 La versión final de este artículo fue realizada por el autor en el ejercicio de la Cátedra Simón Bolívar de la Universidad de Cambridge. 
une radiographie des conditions d'émergence des questions étudiées lors de ces journées. La relation entre culture et démocratisation est abordée dans toute sa complexité, à partir d'une perspective croisant cadre institutionnel et substrat culturel.

Il ébauche de ce fait un cadre socio-politique et culturel pour l'analyse de l'édition indépendante telle quelle est menée dans ce numéro. La production culturelle, loin de constituer un espace isolé de la société, est un élément indispensable et central de la vie démocratique.

Mots Clés: Culture, démocratisation politique, acteurs culturels, transformations culturelles.

Las consideraciones que siguen intentan ser pertinentes a este seminario desde una perspectiva de analista social y político, más que de analista cultural, pero tratando de ver precisamente la dimensión cultural -o lo que creo puede ser la dimensión cultural- de temas tratados más bien en clave politológica o sociológica.

\section{DOS DIMENSIONES DE CULTURA}

Lo primero, al hablar de cultura, es aclarar a qué nos estamos refiriendo. Una definición muy rápida es que hay dos dimensiones: una es la que tiene que ver con los campos, espacios, instituciones y actores culturales, y los grandes aparatos culturales; la cultura, llamémosle "de instituciones". Por otro lado, está la cultura entendida como el sentido individual y colectivo que se da a la vida, al pasado y al futuro, o lo que podríamos llamar la cultura "como sustrato". Así, las políticas culturales tienen esta doble dimensión, aun cuando normalmente se les restringe a la idea de aparatos o instituciones culturales.

La segunda afirmación es que en ambas dimensiones hay una presencia y papel del Estado -aunque las políticas culturales en una sociedad no se reducen a este- $y$ de la sociedad civil. Es cierto que las políticas culturales como aparato en general tienen como actor principal al Estado, pero por supuesto tienen la participación de la sociedad civil. Sin artistas, sin editores independientes, no habría una política cultural en un cierto campo; sin científicos no podría haber política científica, por ejemplo. En cambio, en las políticas de sustrato, el sujeto central es la sociedad civil, los actores sociales, pero evidentemente no puede dejar de haber un rol del Estado. De hecho, lo que se reclama es la ausencia de políticas de Estado frente a los temas de sustrato: por ejemplo el tema de las identidades o de la igualdad o lo que queremos decir cuando hablamos de cultura de derechos humanos. En general, mi impresión -lo voy a decir más adelante- es que frente a políticas culturales institucionales o de aparato relativamente exitosas y que han implicado un cambio en el país, tenemos políticas de sustrato más bien deficitarias. 


\section{LA DEMOCRATIZACIÓN POLÍTICA}

¿De qué se trataba lo que se llamó la transición? Prefiero hablar de democratización política, porque el concepto de transición me parece restringido a un cierto momento o período. Desde mi punto de vista, y lo he sostenido en otros trabajos, la transición en Chile comienza el 5 de octubre de 1988 y termina el 11 de marzo de 1990. Es decir, ese es el período por el cual se pasa de un régimen a otro. Por lo tanto, es mejor hablar, en términos latos, de un proceso de democratización política. Porque si la transición fuera todo el proceso de democratización política todavía estaríamos en transición, y cuando se tiene una transición de veinte años, más larga que el régimen anterior, esto equivaldría al paso del feudalismo al capitalismo o de este al socialismo, lo que toma siglos, y por eso preferimos darle un sentido específico y hablar de democratización política. Voy a referirme, entonces, a estos veinte últimos años.

Si uno puede decirlo muy sintéticamente, la democratización política se trataba por un lado de pasar de una dictadura a un régimen democrático, pero, por otro lado, el caso chileno era el único caso en América Latina en que se heredaba un modelo económico constituido, no una crisis económica que podía resolverse en uno u otro sentido, sino un modelo económico instalado y consolidado. De modo que los componentes de la democratización política, del horizonte, del imaginario, de aquella razón por la cual la gente luchó y se movió, eran, por un lado, derechos humanos, libertades, elecciones libres, y por otro, corrección (en su nivel mínimo), superación (en su nivel máximo) del modelo económico heredado que se definía fundamentalmente en términos de una sociedad de mercado y no solo como economía de mercado. Este modelo significaba imponer el mercado como el modo de convivencia, o como principio funcional central de todas las actividades del país. Y eso tenía como objetivo, como lo ha dicho la OCDE, respecto del modelo educacional, la generación de una sociedad desigual. De eso se trataba el modelo económico y, por lo tanto, se trataba de corregir o superar ese aspecto.

En términos culturales, ¿qué significan estos dos sentidos de la democratización política? La primera significa las libertades y el acceso igualitario, o en igualdad de oportunidades, a los bienes culturales, ese es el componente vinculado a la democratización política, esto es tener un régimen en que se consagran las libertades, y por lo tanto todo lo que ve asociado a ella, en la conformación de sujetos y actores, es decir, temas como la diversidad cultural, ciudadanía cultural, etc. Y la otra, que se corresponde con la corrección o superación del modelo económico, dicho en forma sintética -aunque nadie lo haya dicho así- era pasar del predominio del mercado sobre la cultura, esto es de los bienes culturales concebidos como mercancías al predominio de la cultura, como principio normativo y fuerte de sentido, sobre el mercado. Estas eran entonces las dos dimensiones de la democratización política en términos culturales. $Y$ en este sentido, a la transformación social, económica y 
política, indudable, que hubo en estos últimos veinte años, corresponde también una cierta transformación cultural. No vamos a entrar aquí a la explicación de los fenómenos económicos y sociales. Basta decir que si se pasa de una sociedad de 5 mil USD per cápita a una de 15 mil USD per cápita, hay una transformación fundamental. Si se pasa a una mayoría de niños que nacen en el matrimonio a una sociedad en que la mayoría de los niños nace fuera del matrimonio (no estamos emitiendo un juicio al respecto) estamos en otra sociedad. De una sociedad con un $50 \%$ de pobres a una con $15 \%$ de pobres, hay un salto sideral, independientemente de cómo se mida la pobreza y reconociendo que ella aún es alta. Si uno toma el indicador clásico aunque no único de democracia política, ha habido cinco elecciones presidenciales, otras tantas municipales y parlamentarias y existe un conjunto de libertades públicas garantizadas, aunque no todos coincidan en que esto solo constituye democracia. Las transformaciones en un sentido democrático o de superación del régimen anterior, pueden ser insuficientes, pero su realidad es a mi juicio indiscutible. Que sea una sociedad plenamente democrática o igualitaria eso es otra cosa y lo vamos a abordar más adelante.

\section{LAS TRANSFORMACIONES CULTURALES}

Esta transformación socio-económica y política se corresponde con una cierta transformación cultural. Si analizamos en grueso la dimensión de la cultura en la sociedad chilena, con toda la vaguedad que tiene este concepto, la esfera, el campo cultural, evidentemente hay una profunda transformación. Ello tiene que ver, primero, con que este país generó una institucionalidad para las políticas culturales - débil o no, ese es otro problema- que es unitaria y orgánica, eso es un hecho, y en el campo de la normatividad cultural se hicieron también importantes avances. Aquí yo creo que tal como hay una ley de base de la educación y del medio ambiente, falta una para la cultura, pero se ha avanzado mucho en lo que podríamos llamar la política de aparatos culturales, como por ejemplo, leyes del libro y sobre todo la creación del Consejo Nacional de la Cultura. Aquí ha habido institucionalidad destinada a optimizar el acceso de la gente a los bienes culturales, y por otro lado, focalizada en mejorar o incrementar la innovación y creación científica y artística. Se pueden hacer todas las críticas pertinentes, pero la institucionalidad se generó y eso es extremadamente importante. Pensemos a propósito, guardando las distancias, en la diferencia entre una Francia sin Ministerio de la Cultura y una con: nadie podría negar el cambio, independientemente de si la política gaullista o de Malraux eran buenas o no.

Respecto a la cultura de sustrato, ha habido una expansión y una diversificación, sin duda incompleta, en las demandas de identidades y actores culturales respecto a hace dos décadas. También, si bien estamos en retraso respecto de otras sociedades y 
en algunos temas retrotrayéndonos a los sesenta, es un avance significativo que, por lo menos, el país discuta temas como el aborto y genere leyes y climas de opinión, en los cuales participan también actores culturales, contrarios a cualquier discriminación, especialmente las étnicas y las de orientación sexual o de género. A su vez, gran parte de la demanda por romper un clima de impunidad (y ese es un tema cultural), provino de actores nuevos, no solo políticos, como la Asociación de Familiares de DetenidosDesaparecidos y Ejecutados. En otro campo, los editores independientes constituyen también un actor nuevo en el campo cultural, cuya tarea, fundamentalmente, en la manera en que lo definía Anne Marie Métailié, es orientar la producción cultural y transformarla en un vector de cultura o "Oponerse al mainstring sin ser marginal", como planteaba Paulo Slachevsky.

Entonces, ha habido a lo largo de estos veinte años la aparición de nuevos actores culturales, que plantean nuevos sentidos a la vida colectiva o luchan contra las restricciones a las libertades, logrando terminar con censuras que provenían de la dictadura. Todo ello constituye un avance. Es decir, un conjunto de actores y políticas culturales han permitido que se haya avanzado en la diversificación de la demanda cultural. Culturalmente este país no es el mismo, ni lo es social ni económicamente.

\section{EL ANCLAJE A LA SOCIEDAD DE LA DICTADURA}

Sin embargo, el punto crucial para hacer un balance de estas dos últimas décadas, es que al final la sociedad chilena está todavía amarrada al pasado heredado de la dictadura, por dos anclas fundamentales que tienen, por supuesto, su dimensión y expresión cultural.

La primera de estas dos anclas es el núcleo duro del modelo económico y social, que tiene que ver esencialmente con las desigualdades, y lo que aparece como lo más visible, la punta del iceberg, es la distribución de ingreso y el modelo educacional puesto en relieve por los principales movimientos sociales de los últimos cinco años: el movimiento estudiantil del año 2006 y el movimiento del año 2011, uno escolar y el otro principalmente de la educación superior, aunque también con componentes escolares.

Vale la pena preguntarnos a este respecto, ¿qué es lo que se puso de relieve ahí? Que el problema del país es el tema de la desigualdad. Como indicamos, en el informe de la OCDE de 2004 se dice que la desigualdad no es un efecto del modelo educacional chileno, sino su objetivo buscado. Y lo repitió además la OCDE en el informe el año 2011: el modelo educacional chileno, está construido para generar desigualdades y reproducirlas ampliadamente, situación que es extensible al modelo económico social.

La desigualdad es uno de los componentes de este modelo socioeconómico neoliberal, lo que no es lo mismo que hablar de una sociedad neoliberal, sino una 
sociedad con cruciales componentes neoliberales. Otro de los componentes es la omnipresencia no regulada de poderes fácticos. Los chilenos y chilenas somos víctimas cotidianas de los grandes poderes que antes pertenecían al Estado. ¿Dónde se produce hoy día la máxima explotación (no en el sentido de acumulación de trabajo, explotación en el sentido de opresión, de alienación, de abuso)? Es exactamente en los campos que antes pertenecían al Estado o en el cual el Estado tenía un papel importante: salud con las Isapres, vivienda con las inmobiliarias, previsión con las AFP, todo lo que tiene que ver con las comunicaciones y con educación. Es decir, lo que hay en el país es una enorme concentración de poder económico y mediático frente a una sociedad relativamente inerme al respecto. Y en ese sentido las reformas hechas por todos los gobiernos de la Concertación, tributaria en el caso de Aylwin, modernización del Estado en el caso de Frei, de salud (Auge) en el caso de Lagos, previsión en el caso de Bachelet, implicaron enormes avances, pero todas ellas consolidaron el modelo privatizador y de hegemonía del mercado. Y esa es la primera ancla.

La segunda ancla a la sociedad de la dictadura es que existe, se heredó y se consolidó un sistema institucional para preservar el modelo económico social. Es decir, el modelo político chileno está construido para impedir la transformación del modelo económico social. Este principio de hegemonía del mercado no se puede cambiar porque finalmente quienes lo defienden tienen la capacidad de veto en el sistema político. Y en ese sentido (como han señalado muchos, entre los que me cuento) este es un país donde rige la ley de la minoría. Es la minoría la que pone los temas porque tiene el derecho a veto. Por lo tanto, cuando una minoría tiene derecho a veto en todos los temas sustanciales y además parte con una mitad de los escaños en el parlamento, es esa minoría la que manda. Podrá decirse que también en los sistemas mayoritarios puede llegar a mandar una minoría si busca acuerdos con otros. Pero la diferencia es que no se trata de cualquier minoría. La minoría que hoy además gobierna porque ganó electoralmente, habiendo una contradicción entre lo que podríamos llamar una mayoría sociológica y una mayoría electoral, es directa heredera de la dictadura y, por lo tanto, su tarea es preservar lo logrado en la dictadura. Y tiene por supuesto un representante más fiel en la UDI que impone su proyecto de preservar el sistema político institucional con todo lo valorativo que eso tiene para mantener el modelo económico social, y un representante más díscolo en Renovación Nacional sin proyecto claro, pero que provee liderazgos de apariencia más democrática.

\section{LA SOCIEDAD POSTPINOCHETISTA Y LAS TAREAS PENDIENTES}

Se trata entonces de una democratización incompleta y una corrección del modelo socioeconómico y no de una superación. Eso es lo que permite hablar, a mi juicio, 
de una sociedad post pinochetista. No estamos en una sociedad democrática, estamos en una sociedad post pinochetista, en el sentido que es post autoritaria y no es Pinochet el que manda. Pero las dos anclas a la sociedad pinochetista están ahí y mantienen atada a la sociedad chilena a esa sociedad del pasado.

De modo entonces que la tarea, lo que queda planteado como horizonte histórico, es la superación de la sociedad post pinochetista. Eso significa fundamentalmente, por un lado, devolverle al Estado, al país, a la sociedad, es decir a lo público, el rol dirigente en la economía. Tan simple y tan complejo como eso. Por otro lado, generar una democracia política real significa fundamentalmente un cambio en el sistema institucional, básicamente un cambio en la Constitución. Es decir, una nueva Constitución. Este es el único país del mundo que tuvo una transición o una democratización política y que mantuvo la Constitución heredada de la dictadura, como también el único que tuvo por ocho años al dictador como comandante en jefe del ejército.

Esas son las tareas básicas de la sociedad chilena, independientemente de muchas otras cosas. Todo lo nuevo que se plantea, como la sociedad del conocimiento o ser un país desarrollado sustentablemente, por citar dos temas de la agenda de futuro, está entrampado por esta atadura de la sociedad chilena a la sociedad generada por la dictadura y a la sociedad post pinochetista.

Ahora quisiera señalar, desde una dimensión cultural, algunos de los temas que se presentan dentro de este marco.

\section{DE LA CUltura DE lO POSIBLE A la CULTURA DE PROYECTOS}

En primer lugar, y esto tiene mucho más que ver con una política cultural de sustrato que una política cultural de aparatos culturales, se ha dicho que en Chile la democracia fue una democratización pactada y negociada. No hubo negociación, ninguna. Hubo imposición y adaptación. A eso no se le llama negociación. Luego, no hubo debate y cuando decimos debate, decimos el corazón de una dimensión cultural. Este es un país que no discutió su transición, que tuvo que aceptar lo que a nivel elitario se acordó, pero a ese nivel hubo también imposición y no debate ni negociación verdadera. Lo que se impuso fue un modelo institucional corregido, pero como es difícil aceptar que algo se impuso contra la voluntad del otro, el artefacto explicativo fue el discurso de la negociación, a lo que se le llamó democracia de los consensos o de los acuerdos. Pero para que pueda haber consenso, en el sentido profundo del término, tiene que haber debate y conflicto. No lo hubo sobre ningún tema fundamental: por ejemplo, en los derechos humanos, cuando aparece el informe Rettig sobre Verdad y Reconciliación, lo primero que hace la derecha, los militares y el poder judicial es rechazarlo. No hubo consenso en la organización administrativa 
regional del país y lo están mostrando las manifestaciones regionales de los últimos años como las de Magallanes, Calama y Aysén. No hubo consenso constitucional: todos los proyectos de reforma constitucional para cambiar el sistema electoral han fracasado. Es probable que respecto del tema de reducción de la pobreza hubiera un cierto consenso, pero fue más bien nominal y no trascendió en las políticas. En educación ciertamente hubo imposición y adopción de un modelo que no contaba con ningún consenso excepto entre la derecha y sectores neoliberales y pragmáticos de la Concertación que se encargaron de consolidarlo, marginando las voces críticas como en muchos otros ámbitos.

Se trata, entonces, de pasar de una sociedad regida por la idea de lo posible, (recordemos que la lógica de "en la medida de lo posible" fue implantada en el primer gobierno democrático a propósito del tema de los derechos humanos y se mantuvo a lo largo de todos los gobiernos de la Concertación pese a los cambios de retórica) a una sociedad que quiere quebrar la amarra de lo posible o de la negociación pragmática y reemplazarla por una dinámica del proyecto, de aquello que creemos que es bueno. $\mathrm{Y}$ en ese sentido es muy interesante la frase con la cual los dirigentes estudiantiles definían su movimiento el año 2011: "no queremos mejorar el sistema educacional, queremos reemplazarlo”. Es fácil darse cuenta que eso no está en el campo o la lógica de la posibilidad fáctica, sino de su expansión desde un nuevo horizonte, es decir, la lógica de un proyecto. Entonces, hoy día, estamos mutando desde una cultura de en la medida de lo posible a una cultura de qué queremos para este país. A una cultura de proyectos.

\section{DE LA CULTURA DE LA IMPUNIDAD A LA CULTURA DEL RECONOCIMIENTO Y MEMORIA}

Una segunda cuestión que también ha sufrido una mutación importante es que tuvimos durante diez años, por lo menos, una cultura de la impunidad. La medida de lo posible trasladada al tema de derechos humanos se constituye en impunidad. Y gracias a un juez español se empieza a provocar desde 1997 (pero yo creo que se llega a cristalizar en esa especie de "nunca más" del ejército en 2003) el cambio desde una cultura de la impunidad hacia la posibilidad de una cultura de la justicia. Hoy día no estamos en la vigencia irrestricta de la impunidad como lo estuvimos hasta el 2003, pero sí estamos en una situación de reconocimiento parcial. El paso de la impunidad al reconocimiento a mi juicio ha sido parcial, lo que tiene que ver con que hay un sector de la sociedad que dice que aquí hubo crímenes y asesinatos y hay otro sector de la sociedad que dice no los hubo o que si los hubo fueron necesarios, pero que hay que dar vuelta la hoja. Entonces aquí también hay un problema pendiente porque si uno se da cuenta todos y cada uno de los problemas que el país enfrenta hoy y los problemas futuros están atravesados por las actitudes, por la institucionalidad, por 
las valoraciones que se tiene de lo que fue el golpe militar y el régimen militar. Pero también esto es extensible a otras heridas de la historia del país como es la cuestión mapuche.

Hay ahí también todavía una tarea pendiente: pasar de la cultura de la impunidad a la cultura del reconocimiento y de la reconciliación histórica. La reconciliación histórica implica decir que lo que se hizo nunca debió haberse hecho y sancionar, condenar o castigar todo lo que significó no solo violaciones a los derechos humanos sino sus justificaciones y componentes simbólicos, y ello queda expresado en los textos escolares y en la declaración de principios de la Constitución, como lo han hecho los países que sufrieron el nazismo o fascismo. Hay que recordar que el poder judicial, los medios de comunicación, la Universidad Católica, la UDI, por poner algunos ejemplos, no han hecho este reconocimiento. Estamos todavía a medio camino entre una cultura de impunidad y una cultura de la memoria y del reconocimiento o la reconciliación histórica. E insisto en este último término porque la reconciliación personal es imposible y nadie tiene derecho a exigir que alguien perdone a otro, aunque la reconciliación histórica exija sí pedir perdón como parte del reconocimiento, porque incluso la rabia y el odio de las víctimas forma parte de un proceso de resiliencia y superación de heridas. Lo que importa es la justicia.

En el caso del pueblo mapuche, esto significa no solo reconocimiento de sus demandas, sino actos por los cuales el Estado chileno repara el mal causado y reconoce constitucionalmente la multinacionalidad.

\section{DE LAS LIBERTADES A LOS SUJETOS CULTURALES}

La tercera cuestión, que tiene que ver con la cultura de sustrato, es el paso de las libertades llamemos negativas (o sea la lucha contra las censuras, la lucha y defensa de derechos, la lucha por las libertades) a la constitución de sujetos culturales. Es decir, más allá del clima de respeto a los derechos, la constitución de sujetos que los reclaman.

Esto se puede traducir en dos términos que han sido usados en gran parte por los editores independientes, pero también en el debate más general. Uno es el tema de la ciudadanía cultural y el otro es el tema de la diversidad cultural, ambos son un paso más allá en la lucha por las libertades negativas. Es la dimensión de proyectos no solo a nivel de la sociedad, sino de sujetos, de actores que lo que quieren no es solo que no se les mate, que no se violen sus derechos o que se respeten sus verdades, sino que lo que quieren son espacios de construcción de sus propios proyectos. Yo creo que lo que ocurre, por ejemplo, con actores como el Movilh y otros son una expresión de este tipo de lucha. 


\section{DEL INDIVIDUALISMO A LA SOCIEDAD}

El cuarto gran tema cultural tiene que ver con los sentidos de las movilizaciones y demandas de los últimos tiempos.

Lo que ha habido en el último tiempo es una expansión del concepto o la idea de ciudadano, empoderamiento ciudadano, sociedad civil, etc. Si ello es muy positivo, conlleva, sin embargo, el riesgo de que se desarrolle una cultura de extremo individualismo. Ello puede ocurrir incluso a partir de la doctrina de los derechos humanos cuyos titulares son los individuos, por supuesto que con posterioridad a las luchas contra las violaciones sistemáticas por parte del Estado. Por ejemplo, cuando se confunde el movimiento de protesta de los consumidores abusados con el movimiento estudiantil y se le llama a todo eso "los indignados", yo creo que se comete un error fundamental porque una cosa es la demanda legítima de libertades y de derechos en el plano del consumo y otra es la demanda por cambios en la sociedad. Entonces, el gran tema es cómo se transforma una demanda individual que se ubica fundamentalmente en el plano del consumo (que es una dimensión necesaria y legítima) en demanda ciudadana propiamente tal, pero a su vez, cómo la demanda ciudadana (que siguen siendo individuos) se transforma en demanda de cambio de un campo de la sociedad o de toda ella, en demanda de cambio por una mejor sociedad y no solo en el mejoramiento de la situación individual. El paso del individuo al reconocimiento de la sociedad. En general, a los consumidores explotados o engañados les importa menos el país que su propia situación personal. En cambio, el movimiento estudiantil, el movimiento de Aysén, de lo que están hablando es de sociedad, de país, no solo de la demanda legítima individual. No se trata de volver atrás en materia de demandas individuales. Se ha producido un proceso de individualización indiscutible, pero que también arriesga el narcisismo ciudadano, en el sentido estricto de narciso: un ciudadano que se mira a sí mismo y que termina absorbiéndose y comiéndose porque no entiende que hay una cosa entre medio que se llama la política y la rechaza.

\section{DE LA INSTITUCIONALIDAD CULTURAL AL SUSTRATO}

En síntesis, el gran desafío de la política cultural, como puede concluirse de los cuatro temas señalados, es pasar de una política de institucionalidad a una política de sustrato o, mejor, hacer avanzar la institucionalidad hacia una política de sustrato en la cual, por supuesto, todo lo que tiene que ver con arte y creación mantiene su vigencia y autonomía. Política de sustrato significa que estos temas, y no se trata de temas meramente locales sino que todos ellos tienen una dimensión universal y enraizada en el mundo globalizado, pasan a ser los temas centrales de la política cultural. La gran 
cuestión es cómo se hace esto manteniendo la libertad de creación e innovación. No podemos aquí resolver este problema que queda como un gran desafío. Pero lo que no puede volver a ocurrir es que grandes problemas de la cultura de sustrato (derechos humanos, laicismo, discriminación, diversidad cultural y de género, orientaciones sexuales, aborto, medios de comunicación y televisión pública, medio ambiente, por nombrar solo algunos ejemplos de los últimos años) tengan poco o nada que ver con la institucionalidad y las políticas culturales, que estas queden al margen de las cuestiones de sentido que preocupan a la sociedad y que no provean los espacios de debate y creación en torno a cuestiones que definen lo que un país es o quiere ser.

\section{LA CUESTIÓN CULTURAL DEL SUJETO POLÍTICO-SOCIAL}

En este sentido, el paso de la sociedad post pinochetista a la sociedad democrática a través de las tareas a que nos hemos referido -superar de la hegemonía de mercado y completar una democracia incompleta con una nueva institucionalidad- junto a cuestiones políticas, económicas, estructurales e institucionales, tiene una traducción cultural. Ella consiste, por un lado, en oponer a la hegemonía de mercado la de las orientaciones culturales y a la democracia incompleta la multiplicidad de espacios donde se exprese prácticamente la soberanía popular. Por otro, en el tema de la constitución y orientaciones de quienes encarnan tales tareas, es decir, la cuestión de los sujetos sociales y su modo de constituirse como tales.

Y aquí pareciéramos asistir a una mutación de enorme trascendencia. En Chile, a lo largo del siglo XX el cemento de la sociedad fue la política. Parafraseando a Matta, citado frecuentemente por Bernardo Subercaseaux, lo que hacía que Chile fuera un país más que un paisaje, como lo describe su himno nacional, lo que lo constituía como tal y le daba su identidad era, fundamentalmente, la política. Y las identidades personales y colectivas se definían en la política. No como en el caso argentino o en otros casos a través de una política ligada a un líder o caudillo, sino a través del sistema partidario. El sustrato político cultural era fundamentalmente un sustrato que tenía que ver con identidades generadas y actores constituidos a partir de la política y partidos.

Por ello, los sujetos y los actores sociales fueron siempre políticos sociales o partidarios sociales. No hay un solo movimiento social en Chile (ni cultural hasta digamos los momentos de la democratización y diría que en general hasta el 2006) que no haya sido ligado a partidos políticos. Por lo tanto, el sujeto político de la transformación, el sujeto político social era esta imbricación entre partidos y movimientos. Eso es lo que parece haber cambiado.

Entonces, la gran cuestión es cómo se construye un sujeto tal como el Frente Popular hizo la industrialización, tal como la Democracia Cristiana hizo la reforma 
agraria, tal como la Unidad Popular intentó la vía chilena al socialismo, tal como la alianza democrática, la Concertación y otros sectores, también partidarios y sociales a la vez, hicieron la democratización para esta tarea de la superación de la sociedad post pinochetista, ¿cuál es el sujeto político social?

Y lo que se nos dijo el año 2011 a través del movimiento estudiantil, que ya no podía ser más el sistema de partidos, pero que tampoco, como lo demostró el término de las movilizaciones en ese momento, se puede prescindir del momento político partidario y lo social no puede reemplazar lo partidario.

El gran tema cultural, a mi juicio, es hoy la constitución de un sujeto diversificado que es a la vez político y social. Y en esa problemática, la existencia de actores independientes de partidos, pero con sentido político ( $y$ sin narcicismo que les hace sentir que son ellos los únicos actores porque la clase política y las instituciones son los enemigos), entre ellos los actores culturales, es fundamental. Entre estos actores culturales se encuentran nuestros anfitriones de hoy, los editores independientes, porque juegan con la construcción de orientaciones culturales a partir de un soporte determinado que es el libro, pero que tiene la vinculación principal entre el pasado y la imagen de lo que se quiere en el futuro. Y, además, introduce la cultura en el mundo de la economía que se nos decía era intocable y autónomo.

\section{Referencias bibliográficas}

Garretón, M. (2008). Las políticas culturales en los gobiernos democráticos en Chile. En Rubim, A. y Bayardo, R. (Coords.), Politicas Culturais na Ibero America. Salvador de Bahia: Editora da Universidade Federal da Bahia.

Garretón, M. (2008). Política y sociedad en Chile. Una mirada desde el bicentenario. En Figueroa, M. y Vicuña, M. (Coords.), El Chile del bicentenario. Santiago de Chile: Ediciones Universidad Diego Portales.

Garretón, M. (2010). Sentido, régimen y actores de la política. Cambio, continuidades y perspectivas. En Lagos, R. (Ed.), Cien años de luces y sombras. Santiago de Chile: Taurus. 